\title{
Dual-functional natural-fiber reinforced composites by incorporating magnetite
}

Changlei Xia, ${ }^{b}$ Kaili Wang, ${ }^{a}$ Youming Dong, ${ }^{a}$ Shifeng Zhang, ${ }^{a}{ }^{*}$ Sheldon Q. Shi,${ }^{b, *}$ Liping Cai, ${ }^{b}$ Han Ren, ${ }^{c}$ Hualiang Zhang, ${ }^{c}$ Jianzhang $\mathrm{Li}^{a}{ }^{a}$.

${ }^{a}$ MOE Key Laboratory of Wooden Material Science and Application, Beijing Key Laboratory of Wood Science and Engineering, Beijing Forestry University, Beijing 100083, China

${ }^{b}$ Department of Mechanical and Energy Engineering, University of North Texas, Denton, TX 76203, USA

${ }^{\mathrm{c}}$ Department of Electrical Engineering, University of North Texas, Denton, TX 76203, USA

* Corresponding authors.

Shifeng Zhang: Tel/Fax: +86 1062336072; E-mail: Shifeng.Zhang@bjfu.edu.cn

Sheldon Q. Shi: Tel: +1 9403695930; Fax:+1 9403698675; E-mail: Sheldon.Shi@unt.edu

Jianzhang Li: E-mail: Tel/Fax: +86 1062338083; E-mail: lijianzhang126@126.com

\begin{abstract}
Natural fiber reinforced composites with dual-functional properties, including electromagnetic shielding and paramagnetic functions, as well as improved water-resistant property, were fabricated using vacuum assisted resin transfer molding (VARTM) process. Magnetite particles were firstly mixed with kenaf fibers to form a mat, followed by resin intrusion and compression for the composite fabrication. The obtained kenaf fiber/magnetite/polyester composites were endowed with electromagnetic shielding and paramagnetic properties, without compromising the mechanical properties. When $35.2 \%$ magnetite was incorporated into the composites, the electromagnetic interference shielding effectiveness reached $79.2 \%$ with the range 8 to $12 \mathrm{GHz}$, and the saturation magnetization was $29.2 \mathrm{emu} \mathrm{g}^{-1}$. The 24-hour thickness swelling and water absorption of the composites after introducing magnetite were drastically reduced from $19.7 \%$ and $18.3 \%$ to $2.4 \%$ and $2.6 \%$, counting as the decreases of $87.6 \%$ and $73.4 \%$, respectively. It was indicated that the water resistance of the composite was improved significantly.
\end{abstract}

Keywords: A. Fibres; B. Magnetic properties; E. Resin transfer molding (RTM); Water resistance 


\section{Introduction}

Lignocellulosic fibers have attracted arising interest in composite manufacturing, because of the excellent characteristics such as low density, low cost, ready availability, complete biodegradability, low accumulation in the environment, and successful waste management performance. As one of the lignocellulosic fibers, the application of kenaf (Hibiscus cannabinus, L. Malvaceae) bast fiber was widely investigated due to its lightness, low cost and high specific strength [1-5]. As well known, kenaf is a cellulosic source with both economic and ecological advantages, including the uses of core for natural plywood binder [6] and the bast for soda-AQ pulp [7]. Compared to the energy consumption of the glass fiber, it takes $15 \mathrm{MJ}$ energy to produce $1 \mathrm{~kg}$ kenaf fiber, while, $54 \mathrm{MJ}$ to produce $1 \mathrm{~kg}$ of glass fiber [8]. Furthermore, through the life cycle assessment (LCA), less negative environmental impacts were found in 'cradle to gate' consideration of kenaf fiber compared with the glass fiber [9]. To improve the properties of kenaf composites, many modification efforts were conducted, such as, alkaline treatment [10], rice-washed water treatment [11], using fiber/high-density polyethylene [12], etc. Utilizing an optimal kenaf fiber ratio to achieve the highest tensile property of the composites [13]. The natural fibers, hemp yarn, were used to make tubes [14].

Electromagnetic pollution becomes worldwide preoccupation because of the potentially harmful to human health [15]. Driven by the proliferation of electronics and instrumentation in commercial and industrial applications, electromagnetic interference (EMI) shielding has received arising interest in many areas. In order to reduce or avoid the electromagnetic pollution, the development of EMI shielding material from fully sustainable and eco-friendly materials have become an urgent issue for researchers in the composite industry. Generally, the most commonly used method is to block the field with barriers made of conductive or magnetic materials, so that the energy of electromagnetic wave was reduced to avoid the interference to the working electronic components [16,17]. At the present time, abundant EMI shielding materials and technologies have been produced and designed to reduce the interferences caused by electromagnetic signs [15,18-24]. Among them, metal is the most common EMI shielding material and extensively used in EMI shielding applications [18,19] because of the great electromagnetic reflection. However, the disadvantages of metal are also apparent, such as corrosive action, high density, electric conduction, and uneconomic processing [25]. These drawbacks deter it from the practical applications in the EMI shielding field. To avoid the problems mentioned above, the EMI shielding composites based on the lignocellulosic fibers are considered as the potential candidates [26-30], which are light, corrosion resistant, easily manufactured, economical, and environmentally friendly. Recently, the graphene nanoribbon [31], nano-carbon [22], magnetron sputtering [32] and powdered activated carbon [33] were used to enhance the EMI shielding.

The magnetic composites based on lignocellulosic fibers with both natural fiber properties and magnetic properties were usually prepared by loading magnetic fluids to wood fiber boards using impregnation [34]. The magnetite has been proposed for this application with the advantages of its biocompatibility and easy synthetic process [35]. The fabrication and characterization of magnetic composites based on wood were reported [36-38], which showed that the magnetic wood offered excellent performances such as easy to be heated, electromagnetic wave absorption, paramagnetic, wooden texture, easy to be processed, and humidity control functions. Therefore, magnetic treatment 
endowed the lignocellulosic fiber based composites with new functions and expands their applications. The composites were filled using the graphene oxide-deposited carbon fibers and applied for building materials with high electromagnetic interference shielding [39]. The magnetic wood with electromagnetic wave absorption application as a building material was reported [36].

The magnetite was used to fabricate composites because of the electromagnetic absorbing and paramagnetic properties $[29,40,41]$. The most magnetite was formed in situ from ferric ion and base solution [29,42], which might cause chemical pollution and not be convenient for the industrial production. However, the pollution can be avoided by directly using the magnetite particles. To fabricate the kenaf fiber based composites with electromagnetic shielding and paramagnetic properties, magnetite particles were physically mixed with kenaf fibers. The mixture was fabricated into composite through the vacuum assisted resin transfer molding (VARTM) process, which was expected to improve the mechanical property of composites [43].

In this study, the magnetite-introduced kenaf fiber reinforced composites were manufactured through the VARTM process. The effect of the magnetite contents on the mechanical, dynamic mechanical, water-resistant, electromagnetic shielding, and magnetic properties were investigated.

\section{Materials and Methods}

Sourced from Kengro Corp., USA, the kenaf bast was chopped into approximately $50.8 \mathrm{~mm}$ in length. The sodium hydroxide $(\mathrm{NaOH})$ solution $(5 \%$, w/v) was prepared using $\mathrm{NaOH}$ beads $(\geq 97 \%$, Acros Organics) and deionized (DI) water from the Millipore Milli-Q Integral Water Purification System. The magnetite particles $\left(\mathrm{Fe}_{3} \mathrm{O}_{4}\right)$ were obtained from Beijing Chemical Reagents Co., China, and used as received. The unsaturated polyester resin (AROPOL Q 6585, 30\% styrene, Ashland Chemicals) and tert-butyl peroxybenzoate (t-BP, $98 \%$, Acros Organics) were used for fabricating the composites.

\subsection{Preparation of magnetite loaded fibers}

The kenaf fibers were obtained through the alkali retting process from the kenaf bast. Briefly, a mixture of $120 \mathrm{~g}$ kenaf bast $(9.1 \%$ moisture content measured by Mettler-Toledo HB43-S Moisture Analyzer) and $1.8 \mathrm{~L} \mathrm{NaOH}$ solution were added into a hermetical reactor $(251 \mathrm{M}$, Parr Instrument Company, USA). This alkali retting process was carried out at $160^{\circ} \mathrm{C}$ for one hour with the mechanical stirring. The saturated vapor pressure remained at $0.60 \mathrm{MPa}$ in the hermetical reactor. After cooling, the retted fibers were washed using running water and then dried. The fiber yield was measured as $38.5 \pm 1.0 \%$.

The magnetite loaded fibers were prepared by mixing the retted fibers (after being washed, but not dried) and magnetite. Prior to the mixing, the magnetite aqueous suspension was treated by a VCX 1500 ultrasonic (Sonics \& Materials Inc., USA) for 5 minutes. The retted fibers were mixed into 1-liter magnetite aqueous suspension, and mechanical stirred for 30 minutes. The mixture was formed a preform mat with a dimension of approximate $100 \times 165 \times 10 \mathrm{~mm}$ (width $\times$ length $\times$ thickness), and dried at $105^{\circ} \mathrm{C}$ for 24 hours. The magnetite of $20 \mathrm{~g}\left(20 \mathrm{~g} \mathrm{~L}^{-1}\right), 30 \mathrm{~g}\left(30 \mathrm{~g} \mathrm{~L}^{-1}\right)$, or $40 \mathrm{~g}\left(40 \mathrm{~g} \mathrm{~L}^{-1}\right)$, was fed, and the resulting mats of magnetite loaded fibers were called as Kenaf/magnetite20, Kenaf/magnetite30, or Kenaf/magnetite 40 , respectively. The content of magnetite in each mat was calculated by the weight difference as shown below: 


\section{Magnetite content $=(W$ Kenaf $/$ magnetite $-W$ Kenaf $) / W$ Kenaf $/$ magnetite $\times 100 \%$}

where $W_{\text {Kenaf }} /$ magnetite was the weight of Kenaf/magnetite and $W_{\text {Kenaf }}$ was the weight of retted fiber. The magnetite loading efficiency was calculated as follows:

\section{Loading efficiency $=(W$ Kenaf $/$ magnetite $-W$ Kenaf $) / W$ Feeding magnetite $\times 100 \%$}

where $W_{\text {Feeding magnetite }}$ Was the feeding magnetite, i.e. $20 \mathrm{~g}, 30 \mathrm{~g}$, or $40 \mathrm{~g}$.

\subsection{Morphology analysis}

The Quanta 200 environmental scanning electron microscope (SEM) with an accelerating voltage of 20 $\mathrm{kV}$ and a magnification of 2,000× was used to observe the surfaces of the un-treated kenaf fibers and Kenaf/magnetite40. Prior to the SEM tests, the specimens were coated by a gold sputtering coater for 5 minutes to prevent the charging of the specimens by the SEM electron beam.

\subsection{Determination of magnetite particle size}

The particle-size distribution of magnetite was measured in accordance with dynamic light scattering (DLS) method by Beckman Coulter Delsa ${ }^{\mathrm{TM}}$ Nano C Particle Analyzer. Magnetite was dispersed into the DI-water with a concentration of $1 \mathrm{mg} \mathrm{mL}^{-1}$. Prior to the particle-size measurement, magnetite aqueous dispersion was treated by a VCX 1500 ultrasonic (Sonics \& Materials Inc., USA) for 5 minutes.

\subsection{Fabrication of composites}

The composites were fabricated using the un-treated kenaf fibers, Kenaf/magnetite20, Kenaf/magnetite30, and Kenaf/magnetite40 through vacuum-assisted resin transfer molding (VARTM) process described in the previous research [43]. Briefly, the kenaf preform, with a dimension of $110 \times$ $165 \times 10 \mathrm{~mm}$ (width $\times$ length $\times$ thickness), was placed on the mold that was covered with a flexible polyethylene vacuum bag. The resin (unsaturated polyester resin with $1.5 \%$ of $\mathrm{t}-\mathrm{BP}$ ) was infused by a vacuum pump (Vacmobile 20/2 System with Becker U4.20) with a pressure of 1.3-1.6 $\mathrm{kPa}$. The vacuum force pulled the resin along the distribution layer into the fiber reinforcement preform. The resin-infused preform was cured at $100{ }^{\circ} \mathrm{C}$ for $2 \mathrm{~h}$, and then $150{ }^{\circ} \mathrm{C}$ for $2 \mathrm{~h}$, in the hot press with a pressure of $13 \mathrm{MPa}$. Once the fabricated composite cooled down to room temperature, the vacuum bag and distribution layer were removed.

\subsection{Mechanical property tests}

From each composite, twelve specimens sized $25 \times 160 \times 3 \mathrm{~mm}$ (width $\times$ length $\times$ thickness) were cut by a 9-in Skil benchtop band saw (Robert Bosch Tool Corp., USA), for examining the modulus of elasticity (MOE), modulus of rupture (MOR), and bending elongation at break (BEB) of the composites using the Shimadzu AGS-X universal testing machine in accordance with the procedure described in ASTM D790 standard. Three-point bending set-up was used with a span of $50 \mathrm{~mm}$ and a crosshead 
speed of $1.3 \mathrm{~mm} \mathrm{~min}^{-1}$. The tensile modulus (TE), tensile strength (TS), and tensile elongation at break (TEB) of the specimens (twelve replicates) were tested in accordance with the procedures described in ASTM D638 standard by the Shimadzu AGS-X tester with an axial extensometer (Epsilon Technology Corp., USA) with a $50.8 \mathrm{~mm}(2 \mathrm{inch})$ gauge length. The specimen dimensions were $19 \times 165 \times 3 \mathrm{~mm}$ (width $\times$ length $\times$ thickness) and the cross section of the narrow section was $13 \times 57 \mathrm{~mm}$ (width $\times$ length). A crosshead speed of $5 \mathrm{~mm} \mathrm{~min}^{-1}$ was used.

\subsection{Dynamic mechanical analysis}

A TA instruments Q800 DMA tester was employed for the dynamic mechanical analysis (DMA). Each specimen sized $1 \times 4 \times 30 \mathrm{~mm}$ (thickness $\times$ width $\times$ length) was used for the DMA three-point bending tests with a gauge length of $25.4 \mathrm{~mm}$. The temperature from 35 to $200{ }^{\circ} \mathrm{C}$ with a ramping speed of $5{ }^{\circ} \mathrm{C}$ $\min ^{-1}$ was performed, and the frequency of the oscillation was set to be $1 \mathrm{~Hz}$. The storage modulus (E'), loss modulus (E") and mechanical loss factor $(\tan \delta)$ were plotted as a function of temperature.

\subsection{Water-resistant tests}

The composites made with un-treated and magnetite loaded fibers were used to determine their thickness swelling and water absorption. The specimens with a dimension of $25 \times 25 \times 3 \mathrm{~mm}$ (width $\times$ length $\times$ thickness) were cut using the 9-in Skil benchtop band saw. Five replicates were tested for each composite. Twenty four-hour water submersion tests were carried out in accordance with the ASTM D1037 standard for the determination of the thickness swelling and water absorption.

\subsection{Electromagnetic interference shielding tests}

The electromagnetic interference shielding effectiveness (EMI SE) measurement with both amplitude and phase properties was carried out using an E8363B PNA Network Analyzer (Agilent Technologies Inc., USA) in a frequency ranging from $8 \mathrm{GHz}$ to $12 \mathrm{GHz}$, presenting the results of the shielding measurements in decibels $(\mathrm{dB})$. All the specimens were machined to the same dimension $(42 \times 42 \mathrm{~mm})$ with the corresponding WR90 SMA/F waveguides (Fairview Microwave Inc., USA), which led to the samples exactly fit the size of waveguides. The mode of measurement was a typical transmission measurement (scalar $\mathrm{S}_{21}$ - measurement). The $\mathrm{dB}$ value described the level of the incident power or power-flux density decreased after it passed the device during the test. To calculate the $\mathrm{dB}$ value from the incident power $P_{1}$, respectively, from the arriving electrical field strength $E_{1}$ and the transmitted power $P_{2}$ or field strength $E_{2}$, the following equation was used:

$$
S E \mathrm{~dB}=10 \log (P 1 P 2)=20 \log (E 1 E 2)
$$

The received $\mathrm{dB}$ values were then converted into percentage of EMI SE using the following equation:

$$
S E=1-10-S E \mathrm{~dB} 10 \times 100 \%
$$

\subsection{Magnetic tests}

Magnetic hysteresis loops of the specimens were determined with a 7410 VSM meter (Lake Shore Cryotronics, Inc., USA) at $300 \mathrm{~K}$ in an external magnetic field from -20 to $20 \mathrm{kOe}$ in 100 Oe steps and 
an averaging time of $100 \mathrm{~ms}$. The coercivity $\left(H_{\mathrm{c}}\right)$, saturation magnetization $\left(M_{\mathrm{s}}\right)$, and retentivity $\left(M_{\mathrm{r}}\right)$ were also evaluated. The dimensions of specimens for magnetic tests were $3 \times 7 \times 3 \mathrm{~mm}$ (width $\times$ length $\times$ thickness). The specific $M_{\mathrm{s}}$ of the composites was calculated as following:

$$
\text { Specific } M s=M s \text { Magnetite content }
$$

\section{Results and Discussion}

\subsection{Characterization of kenaf fibers}

The components of the un-treated Kenaf, Kenaf/magnetite20, Kenaf/magnetite30, and Kenaf/magnetite40, are presented in Table 1. The magnetite loading efficiency was calculated according to Eq. (1). The results showed that, with the increase of feeding magnetite from $20 \mathrm{~g}$ to $30 \mathrm{~g}$ and $40 \mathrm{~g}$, the composition of magnetite was increased from $31.0 \%$ to $39.5 \%$ and $44.3 \%$, respectively. However, the loading efficiency, calculated by Eq. (2), was slightly reduced from $86.4 \%$ to $83.8 \%$ and $76.7 \%$, respectively, with the increase of feeding magnetite.

Table 1. Components of un-treated and treated kenaf fibers.

\begin{tabular}{llllll}
\hline \multirow{2}{*}{ Specimen } & \multicolumn{2}{c}{ Magnetite } & & \multicolumn{2}{c}{ Components (wt.\%) } \\
\cline { 2 - 3 } \cline { 6 - 7 } & Feeding (g) & Loading efficiency (\%) & & Fiber & Magnetite \\
\hline Kenaf & - & - & & 100.0 & - \\
Kenaf/magnetite20 & 20 & 86.4 & & 69.0 & 31.0 \\
Kenaf/magnetite30 & 30 & 83.8 & & 60.5 & 39.5 \\
Kenaf/magnetite40 & 40 & 76.7 & & 55.7 & 44.3 \\
\hline
\end{tabular}

The SEM images of the Kenaf and Kenaf/magnetite40 are shown in Fig. 1 for comparison. The magnetite particles on the fiber surface were visible in Kenaf/magnetite40 (Fig. 1b), compared with untreated Fiber (Fig. 1a). The particle-size distribution of the magnetite is shown in Fig. 2. The numberbased (Fig. 2a) and volume-based (Fig. 2b) distributions of magnetite particle size showed that the magnetite had peaks at $159.7 \pm 51.3 \mathrm{~nm}$, and $254.1 \pm 180.5 \mathrm{~nm}$, respectively.

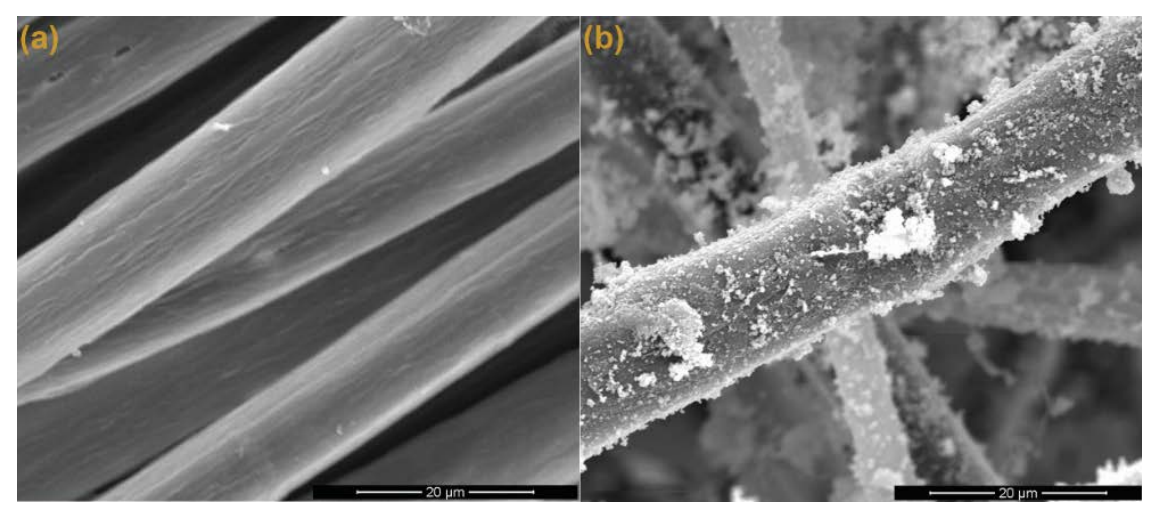

Fig. 1. SEM observation of the Kenaf (a), Kenaf/magnetite40 (b. 

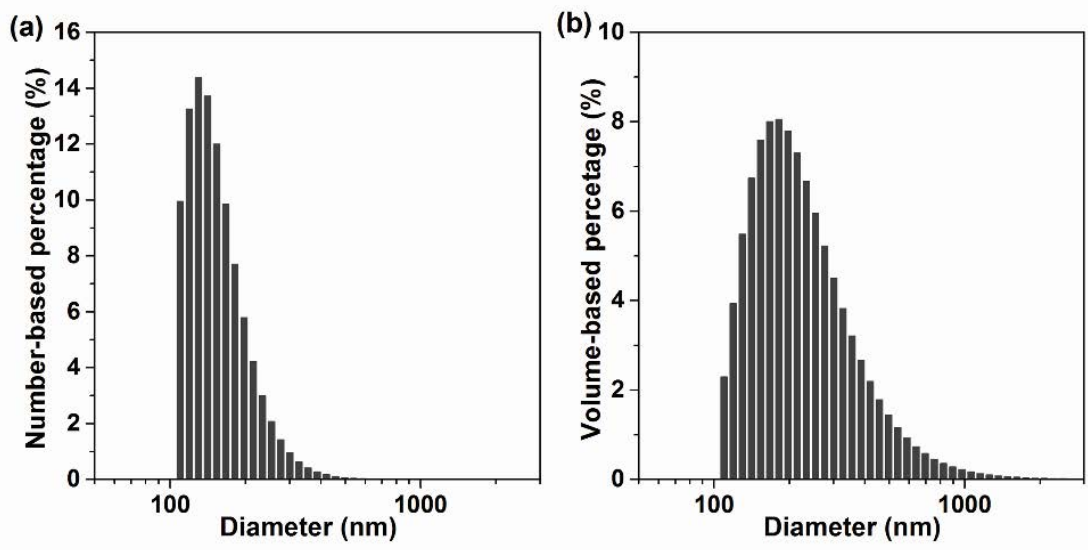

Fig. 2. Particle-size distribution of magnetite in differential number (a), and differential volume (b).

\subsection{Mechanical properties of composites}

The physical properties of the composites are summarized in Table 2. The resin content of Kenaf/polyester was $33.3 \%$, which was reduced by $20.7-25.4 \%$ after the magnetite loaded fibers were used, and also the fiber content was reduced from $66.7 \%$ to $44.1-53.2 \%$. The density was increased from 1,159.4 $\mathrm{Kg} \mathrm{m}^{-3}$ (Kenaf/polyester), to 1,473.4 $\mathrm{Kg} \mathrm{m}^{-3}$ (Kenaf/magnetite20/polyester), 1,600.1 $\mathrm{Kg} \mathrm{m}^{-}$

${ }^{3}$ (Kenaf/magnetite30/polyester), and 1,609.2 $\mathrm{Kg} \mathrm{m}^{-3}$ (Kenaf/magnetite40/polyester), respectively. Since the magnetite has a density between 4.8 to $5.1 \mathrm{~g} \mathrm{~cm}^{-3}$, the densities of the composites increased dramatically after the magnetite particles being introduced.

Table 2. Physical and water-resistant properties of the composites.

\begin{tabular}{|c|c|c|c|c|c|c|}
\hline \multirow[t]{2}{*}{ Composite } & \multirow{2}{*}{$\begin{array}{l}\text { Density } \\
\left(\mathrm{Kg} \mathrm{m}^{-3}\right)\end{array}$} & \multicolumn{3}{|c|}{ Components (wt. \%) } & \multicolumn{2}{|c|}{ 24-hour water submission (\%) } \\
\hline & & Resin & Fiber & Magnetite & Thickness swelling & Water absorption \\
\hline Kenaf/polyester & $1,159.4(44.5)^{\mathrm{a}}$ & 33.3 & 66.7 & - & $19.7(3.8)$ & $18.3(2.3)$ \\
\hline Kenaf/magnetite20/polyester & $1,473.4(61.3)$ & 23.0 & 53.2 & 23.9 & $5.0(1.4)$ & $4.9(0.7)$ \\
\hline Kenaf/magnetite $30 /$ polyester & $1,600.1(42.0)$ & 25.4 & 45.2 & 29.5 & $2.5(0.6)$ & $2.7(0.2)$ \\
\hline Kenaf/magnetite $40 /$ polyester & $1,609.2(79.5)$ & 20.7 & 44.1 & 35.2 & $2.4(0.3)$ & $2.6(0.2)$ \\
\hline
\end{tabular}

Note: ${ }^{\mathrm{a}}$ mean (standard deviation)

The results of 3-point bending tests (MOE, MOR and BEB) of the four types of composites are compared in Fig. 3. By adding the magnetite, the composite MOEs were increased by $50.1 \%$ (Kenaf/magnetite20/polyester composite), 52.9\% (Kenaf/magnetite30/polyester composite), and 46.6\% (Kenaf/magnetite40/polyester composite). The MORs of the Kenaf/magnetite20/polyester, Kenaf/magnetite30/polyester, and Kenaf/magnetite40/polyester composites were by $75.3 \%, 60.6 \%$, and $43.6 \%$, respective. In addition, BEBs of the Kenaf/magnetite20/polyester, Kenaf/magnetite30/polyester, 
and Kenaf/magnetite40/polyester composites were slightly increased by $29.5 \%, 13.7 \%$, and $2.5 \%$, respectively. In summary, the addition of magnetite could help improving the anti-flexural properties. In a report [29] of magnetite loaded kenaf fiber reinforced composites fabricated using the traditional hotpressing method, MOE was decreased from 2,875 $\mathrm{MPa}$ to 2,729 - 2,007 $\mathrm{MPa}$ after introducing magnetite particles. However, in this work the MOE was significantly increased. It might be contributed to the VARTM process, which could enhance MOE considerably compared with the traditional hotpressing process [43].
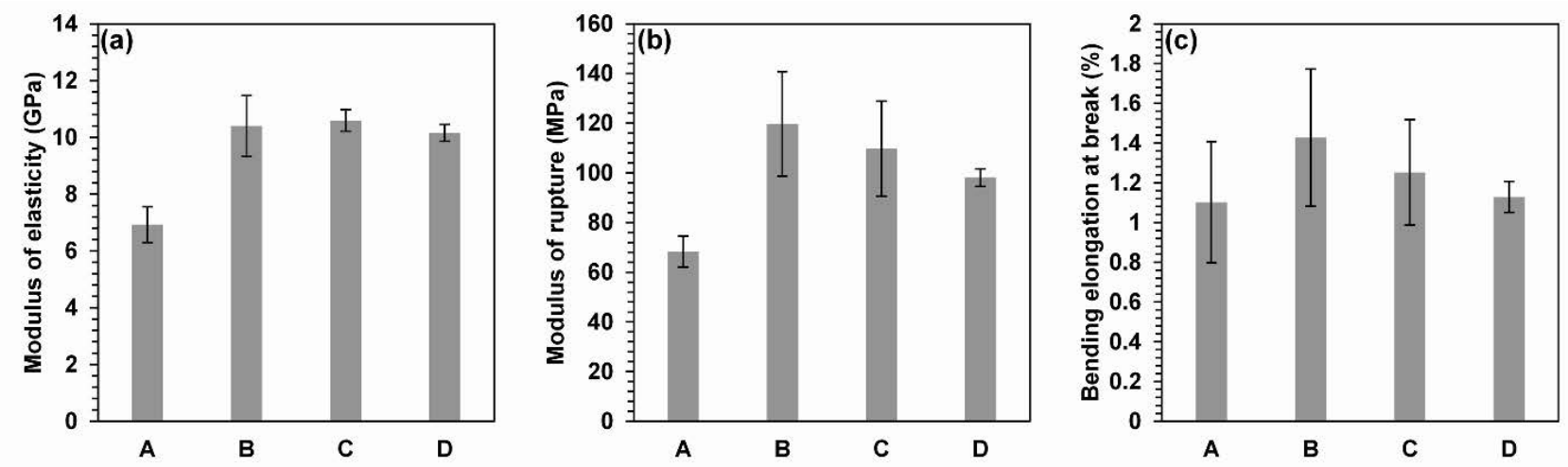

Fig. 3. Modulus of elasticity (a), modulus of rupture (b) and bending elongation at break (c) from the 3point bending tests of the four composites including Kenaf/polyester (A), Kenaf/magnetite20/polyester (B), Kenaf/magnetite30/polyester (C), and Kenaf/magnetite40/polyester (D).

TE, TS, and TEB of the four types of composites are compared in Fig. 4. The TE of Kenaf/magnetite20/polyester, Kenaf/magnetite30/polyester, and Kenaf/magnetite40/polyester composites were increased by $37.7 \%, 18.0 \%$, and $14.7 \%$, respectively, compared with the Kenaf/polyester composite of $10.7 \pm 1.0 \mathrm{GPa}$. Although TS of Kenaf/magnetite40/polyester composites $(24.8 \pm 3.0 \mathrm{MPa})$ were reduced by $44.3 \%$, the other types of composites were comparable with the Kenaf/ polyester composite $(44.5 \pm 3.7 \mathrm{MPa})$. The TEB of Kenaf/magnetite20/polyester $(1.48 \pm 0.79 \%)$, Kenaf/magnetite30/polyester $(1.28 \pm 0.42 \%)$ composites were increased by $30.2 \%$ and $12.7 \%$, compared with the Kenaf/polyester composite $(1.14 \pm 0.50 \%)$. The same as TS, the TEB of Kenaf/magnetite $40 /$ polyester composite $(0.69 \pm 0.11 \%)$ was decreased by $39.1 \%$. In summary, the tensile properties of Kenaf/magnetite20/polyester and Kenaf/magnetite30/polyester composites are comparable or excessed those of the Kenaf/polyester composite. However, with highest content of magnetite, the tensile properties of Kenaf/magnetite40/polyester composite decreased. 

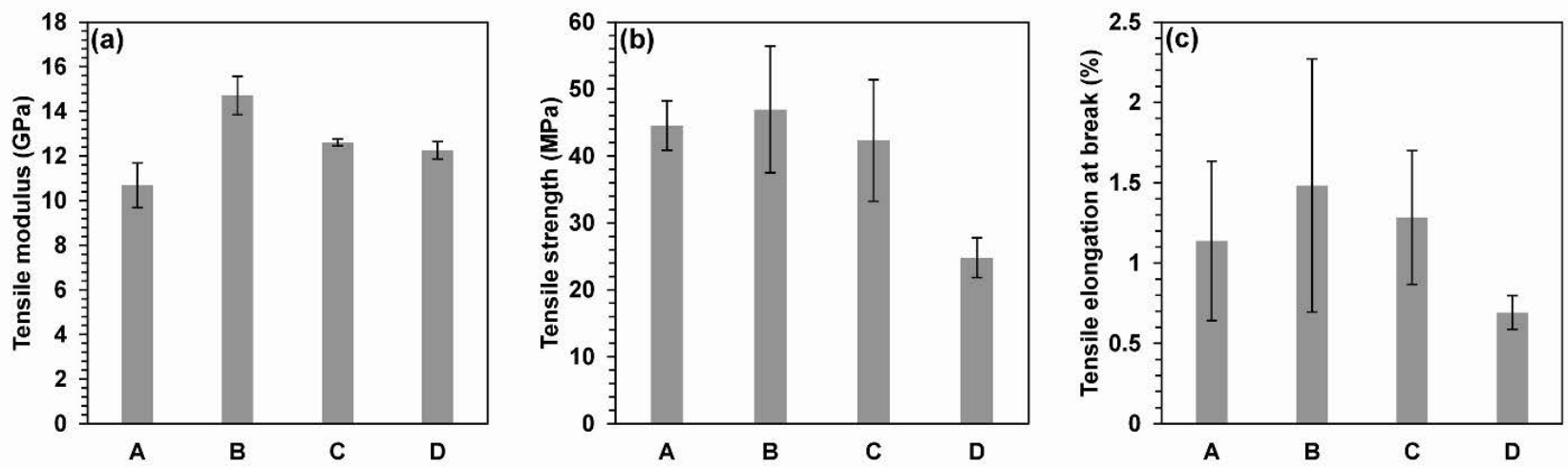

Fig. 4. Tensile modulus (a), tensile strength (b) and tensile elongation at break (c) from the tensile tests of the four composites including Kenaf/polyester (A), Kenaf/magnetite20/polyester (B), Kenaf/magnetite30/polyester (C), and Kenaf/magnetite40/polyester (D).

\subsection{Dynamic mechanical properties of composites}

Storage modulus is an index of resilience that measures the energy storage capability of material after elastic deformation. Fig. 5a shows the $\mathrm{E}^{\prime}$ of the four composites. In general, the $\mathrm{E}^{\prime}$ of the four composites decreased as temperature increased because of the increasing chain mobility of the polymer matrix [44]; the E' of the composites with additional magnetite were much higher than Kenaf/polyester composite; and the $\mathrm{E}^{\prime}$ of the composites decreased with the increase of magnetite content. It was similar to the mechanical properties (TE, TS and MOR), indicating that magnetite particles were able to help improving both static and dynamic mechanical properties. It's easy to find the peaks on the $E^{\prime \prime}$ and tan $\delta$ curves for Kenaf/polyester composite (Fig. 5b-c), i.e. $108.5^{\circ} \mathrm{C}$ for E", and $113.4{ }^{\circ} \mathrm{C}$ for tan $\delta$, however, no clear peaks for the composites with additional magnetite were observed. No unambiguous glass transition temperatures for the magnetite introduced composites were detected from DMA tests (Fig. 5bc), which might result from the improved interaction between the components of the composites $[45,46]$. It was indicated that the addition of magnetite reduced the chain mobility of the polyester matrix, so that the mechanical properties were enhanced at high temperatures.
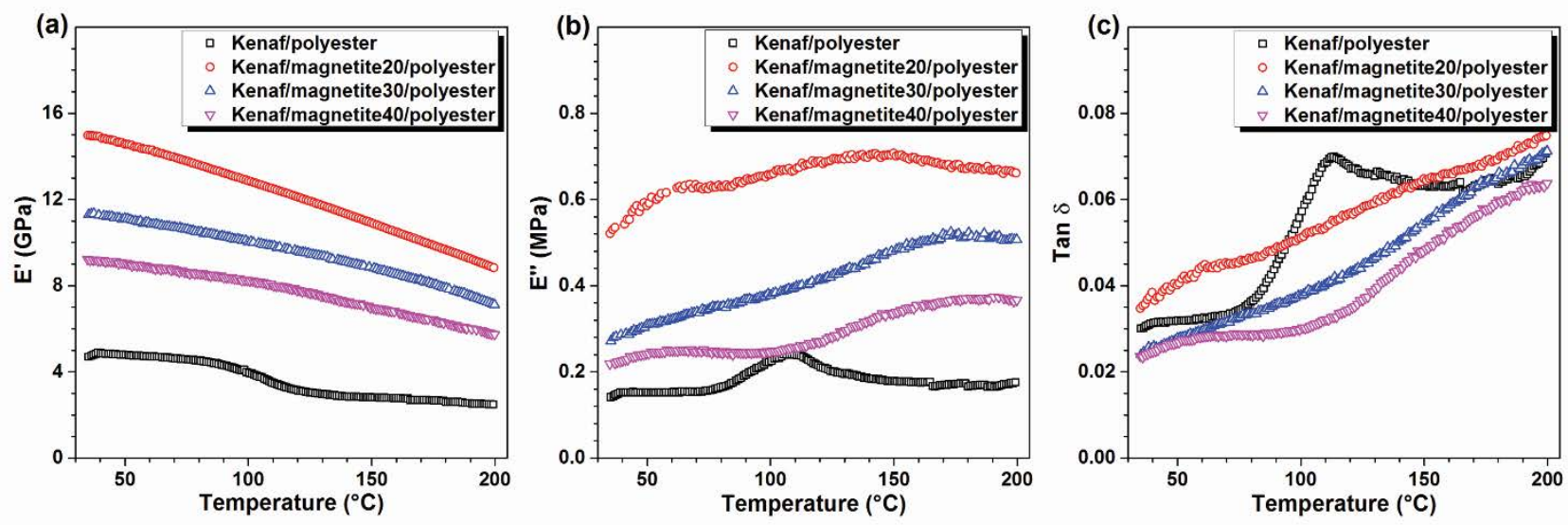

Fig. 5. DMA results of the four composites, including storage modulus (a), loss modulus (b), and damping parameter (c). 


\subsection{Water-resistant properties of composites}

The results from 24-h water submission of the four composites, including thickness swelling and water absorption, are presented in Table 2. Compared with the Kenaf/polyester composite (19.7\%), the thickness swellings of Kenaf/magnetite20/polyester, Kenaf/magnetite30/polyester, and Kenaf/magnetite40/polyester composites were 5.0\%, 2.5\%, and $2.4 \%$, which showed reductions of $74.4 \%, 87.1 \%$, and 87.6\%, respectively. The statistical analysis (ANOVA, $\alpha=0.001$ ) showed that the reductions in thickness swelling of the magnetite modified Kenaf/polyester composites were significant at the level of $\alpha=0.001$. The same trends were found for the water absorptions, in which the Kenaf/magnetite20/polyester $\quad(4.9 \%), \quad$ Kenaf/magnetite30/polyester $\quad(2.7 \%), \quad$ and Kenaf/magnetite40/polyester (2.6\%) composites were significantly (ANOVA, $\alpha=0.001$ ) decreased by $73.4 \%, 85.4$, and $85.7 \%$, respectively, compared with that of Kenaf/polyester composite (18.3\%). In the previous report [47], the 24-hour water absorption of the glass-fiber sheet molding compound (SMC) was determined to be $1.1 \%$. Compared to the Kenaf/magnetite40/polyester composite with glass-fiber $\mathrm{SMC}$, although the 24-hour water absorption was slightly higher (2.6\% vs. $1.1 \%)$, the water-resistant property of the Kenaf/magnetite40/polyester composite performed excellent, in considering the less hydrophobic resin contents, glass-fiber SMC (20.7\%) vs. Kenaf/magnetite40/polyester (26.0\%), and the mineral contents $(35.2 \%$ vs. $46.0 \%)$ [47].

In summary, the water-resistant properties of the composites were dramatically improved by adding the magnetite. Owing to the hydrogen bonding between magnetite and cellulosic fibers, magnetite adhered to the fiber surfaces and blocked the large amount of hydroxyl groups. The magnetite dramatically reduced the amount of hydroxyl groups on the fiber surface, resulting in better water resistance. The phenomena that deposited particles on the fiber surfaces block the hydroxyl groups resulting an improvement of the water-resistance could also be found when calcium carbonate particles were used $[47,48]$.

\subsection{Electromagnetic interference shielding properties of composites}

The results of EMI SE at the range of 8-12 GHz for the four types of composites are compared in Fig. 6. The average SEs of the Kenaf/polyester, Kenaf/magnetite20, Kenaf/magnetite30, and Kenaf/magnetite40 composites were $41.4 \pm 5.5 \%, 64.4 \pm 7.6 \%, 77.0 \pm 3.9 \%$, and $79.2 \pm 3.9 \%$, respectively. Compared to the SE of Kenaf/polyester composite (41.4\%), SE of the composites after using Kenaf/magnetite20, Kenaf/magnetite30, and Kenaf/magnetite40 were increased to be $64.4 \%$, $77.0 \%$, and $79.2 \%$, respectively. In Fig. 6, it was also found that, although the composite SE increased with the magnetite content, it was almost independent of frequency in the measured frequency range. The results showed that, generally, the larger the magnetite content, the higher the value of EMI SE was obtained. However, the increase of EMI SE showed a limitation in Fig.5. When the magnetite content was increased from $29.5 \%$ (the Kenaf/magnetite30/polyester composite) to $35.2 \%$ (the Kenaf/magnetite40/polyester composite), the both composites showed the approximately similar EMI SE values. 


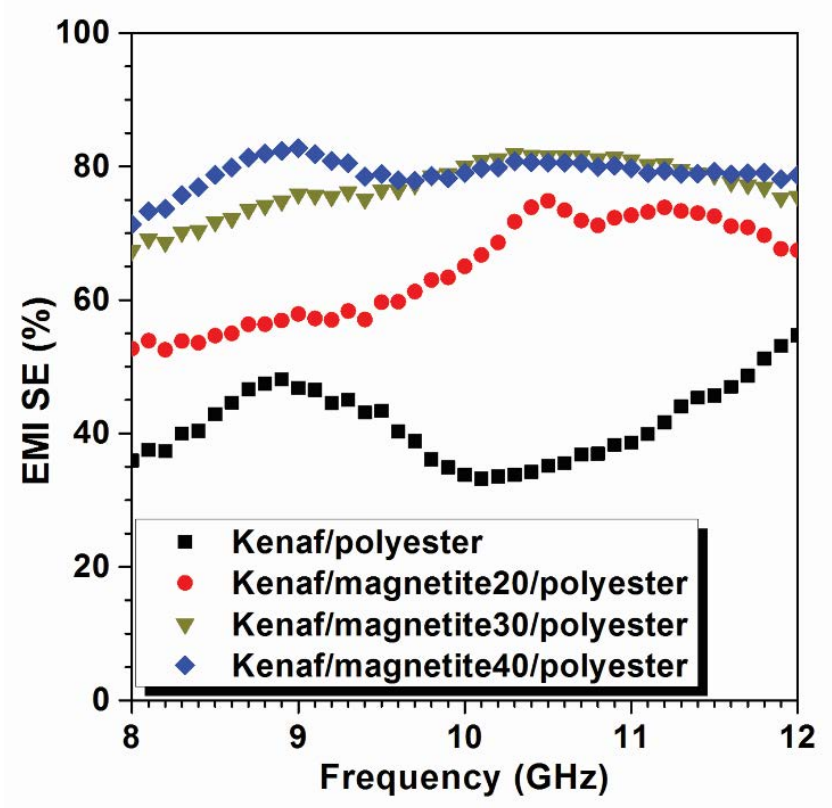

Fig. 6. Electromagnetic interference shielding effectiveness of the four composites.

\subsection{Magnetic properties of composites}

Fig. 7 shows the magnetic hysteresis curves of the four composites measured with the VSM meter, and the results of $H_{\mathrm{c}}, M_{\mathrm{s}}$, and $M_{\mathrm{r}}$ are presented in Table 3. The magnetic hysteresis curves showed that the Kenaf/magnetite/polyester composites exhibited a clear hysteretic behavior but not for the Kenaf/polyester composite. Regarding $H_{\mathrm{c}}$ and $M_{\mathrm{r}}$, the Kenaf/magnetite/polyester composites showed negligible coercivity and remanence, confirming the superparamagnetic properties [40,49]. The $M_{\mathrm{s}}$ of the Kenaf/magnetite20/polyester, Kenaf/magnetite30/polyester, and Kenaf/magnetite40/polyester composites were 19.3, 25.5, and $29.2 \mathrm{emu} \mathrm{g}^{-1}$, respectively, which showed a gradual enhancement with the increase in magnetite content. However, the values of specific $M_{\mathrm{s}}$ of magnetic composites (81.1, 86.4 , and $83.0 \mathrm{emu} \mathrm{g}^{-1}$, respectively) were smaller than that of the bulk magnetite ( $\left.93 \mathrm{emu} \mathrm{g}^{-1}\right)$ [50]. The reason could be that the composite structure probably affects the magnetic properties of magnetic particles [41]. 


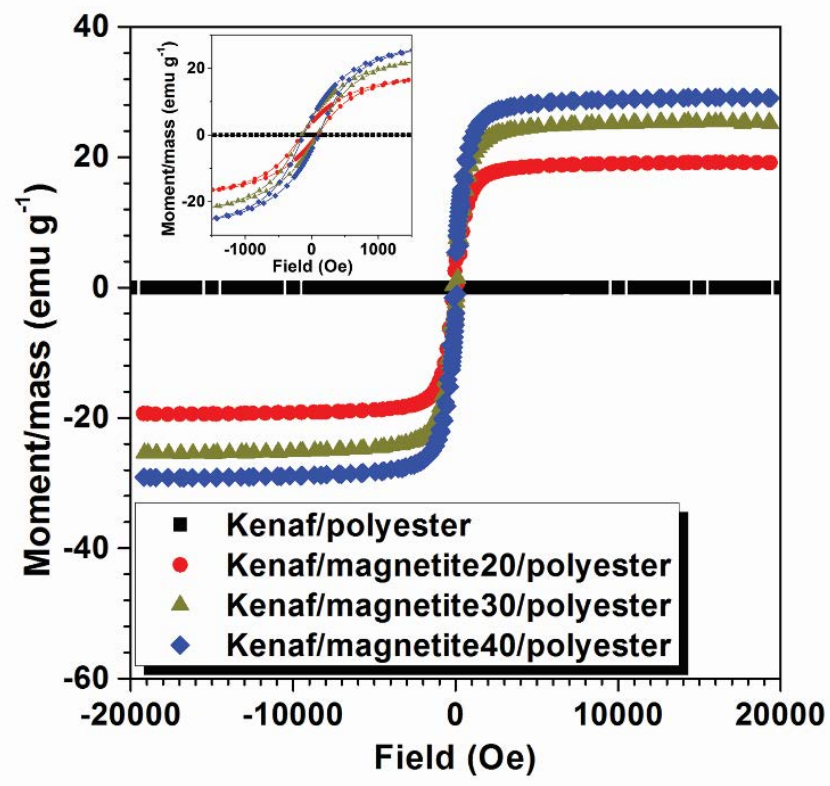

Fig. 7. Magnetic hysteresis curves of four composites.

Table 3. Coercivity, saturation magnetization, and retentivity of the four composites.

\begin{tabular}{lllll}
\hline Composite & $\begin{array}{l}H_{\mathrm{c}}{ }^{\mathrm{a}} \\
(\mathrm{Oe})\end{array}$ & $\begin{array}{l}M_{\mathrm{r}}{ }^{\mathrm{b}} \\
\left(\mathrm{emu} \mathrm{g}^{-1}\right)\end{array}$ & $\begin{array}{l}M_{\mathrm{s}}{ }^{\mathrm{c}} \\
\left(\mathrm{emu} \mathrm{g}^{-1}\right)\end{array}$ & $\begin{array}{l}\text { Specific } M_{\mathrm{s}}{ }^{\mathrm{d}} \\
\left(\mathrm{emu} \mathrm{g}^{-1}\right)\end{array}$ \\
\hline Kenaf/polyester & - & 0.0021 & 0.0191 & - \\
Kenaf/magnetite20/polyester & 115.8 & 3.0 & 19.3 & 81.1 \\
Kenaf/magnetite30/polyester & 119.5 & 4.1 & 25.5 & 86.4 \\
Kenaf/magnetite40/polyester & 112.2 & 4.8 & 29.2 & 83.0 \\
\hline
\end{tabular}

Notes: ${ }^{\mathrm{a}} H_{\mathrm{c}}=$ coercivity; ${ }^{\mathrm{b}} M_{\mathrm{r}}=$ retentivity; ${ }^{\mathrm{c}} M_{\mathrm{s}}=$ saturation magnetization; ${ }^{\mathrm{d}}$ Specific $M_{\mathrm{s}}$ was calculated in accordance with Eq. (5).

\section{Conclusions}

Kenaf fiber reinforced polyester composites were successfully fabricated through the VARTM process. The composites were endowed with electromagnetic shielding and paramagnetic properties by introducing magnetite. After introducing 23.9\%, 29.5\% and 35.2\% magnetite into the composites, the electromagnetic shielding, paramagnetic, and water-resistant properties of the composites were significantly improved while the mechanical properties were not compromised except the tensile property of the composite with the addition of 35.2\% magnetite. The following conclusions were drawn from this study: (1) the electromagnetic interference shielding effectiveness was increased from $41.4 \%$ to $64.4 \%, 77.0 \%$ and $79.2 \%$; (2) the saturation magnetization was increased from $0.0191 \mathrm{emu} \mathrm{g}^{-1}$ to $19.3,25.5$ and 29.2 emu g $^{-1}$; (3) the 24 -h thickness swelling was reduced from $19.7 \%$ to $5.0 \%, 2.5 \%$ and $2.4 \%$, and the $24-\mathrm{h}$ water absorption was reduced from $18.3 \%$ to $4.9 \%, 2.7 \%$ and $2.6 \%$.

\section{Acknowledgments}


This research was supported in part by National Science Foundation (NSF) CMMI 1247008, Fundamental Research Funds for the Central Universities (NO.TD2011-12), Beijing Natural Science Foundation (Project 2151003), and National Natural Science Foundation of China (Project 31000268/C160302). 


\section{References}

[1] Liang K, Shi SQ, Nicholas DD, Sites L. Accelerated weathering test of kenaf fiber unsaturated polyester sheet molding compounds. Wood Fiber Sci 2013;45(1):42-48.

[2] Xia C, Shi SQ, Cai L, Nasrazadani S. Increasing inorganic nanoparticle impregnation efficiency by external pressure for natural fibers. Ind Crop Prod 2015;69:395-399.

[3] Jonoobi M, Harun J, Tahir PM, Shakeri A, SaifulAzry S, Makinejad MD. Physicochemical characterization of pulp and nanofibers from kenaf stem. Mater Lett 2011;65(7):1098-1100.

[4] Zaini LH, Jonoobi M, Tahir PM, Karimi S. Isolation and characterization of cellulose whiskers from kenaf (Hibiscus cannabinus L.) bast fibers. J Biomater Nanobiotechnol 2013;4:37.

[5] Xia C, Zhang S, Shi SQ, Cai L, Huang J. Property enhancement of kenaf fiber reinforced composites by in situ aluminum hydroxide impregnation. Ind Crop Prod 2016;79:131-136.

[6] Okuda N, Sato M. Finely milled kenaf core as a natural plywood binder. Holzforschung 2007;61(4):439-444.

[7] Feng ZA, Alen R, Niemela K. Formation of aliphatic carboxylic acids during soda-AQ pulping of kenaf bark. Holzforschung 2002;56(4):388-394.

[8] Akil HM, Omar MF, Mazuki AAM, Safiee S, Ishak ZAM, Abu Bakar A. Kenaf fiber reinforced composites: A review. Mater Design 2011;32(8-9):4107-4121.

[9] Wang J, Shi SQ, Liang K. Comparative Life-cycle Assessment of Sheet Molding Compound Reinforced by Natural Fiber vs. Glass Fiber. J Agric Sci Technol B 2013;3:493-502.

[10] Fiore V, Di Bella G, Valenza A. The effect of alkaline treatment on mechanical properties of kenaf fibers and their epoxy composites. Composites Part B 2015;68:14-21.

[11] Park J, Choi J, Wang Z, Kwon D, Shin P, Moon S, DeVries KL. Comparison of mechanical and interfacial properties of kenaf fiber before and after rice-washed water treatment. Composites Part B 2015;83:21-26.

[12] Salleh FM, Hassan A, Yahya R, Azzahari AD. Effects of extrusion temperature on the rheological, dynamic mechanical and tensile properties of kenaf fiber/HDPE composites. Composites Part B 2014;58:259-266.

[13] Kwon H, Sunthornvarabhas J, Park J, Lee J, Kim H, Piyachomkwan K, Sriroth K, Cho D. Tensile properties of kenaf fiber and corn husk flour reinforced poly(lactic acid) hybrid bio-composites: Role of aspect ratio of natural fibers. Composites Part B 2014;56:232-237.

[14] Weclawski BT, Fan M, Hui D. Compressive behaviour of natural fibre composite. Composites Part B 2014;67:183-191. 
[15] Huynen I, Quievy N, Bailly C, Bollen P, Detrembleur C, Eggermont S, Molenberg I, Thomassin JM, Urbanczyk L, Pardoen T. Multifunctional hybrids for electromagnetic absorption. Acta Mater 2011;59(8):3255-3266.

[16] Koh Y, Sambasevam KP, Yahya R, Phang S. Improvement of microwave absorption for PAni/HA/TiO2/Fe3O4 nanocomposite after chemical treatment. Polym Composite 2013;34(7):11861194.

[17] Li X, Yi H, Zhang J, Feng J, Li F, Xue D, Zhang H, Peng Y, Mellors NJ. Fe3O4-graphene hybrids: nanoscale characterization and their enhanced electromagnetic wave absorption in gigahertz range. $\mathrm{J}$ Nanopart Res 2013;15(3):1472.

[18] Hou Cui-ling, Li Tie-hu, Zhao Ting-kai, Liu He-guang, Liu Le-hao, Zhang Wen-juan. Electromagnetic wave absorbing properties of multi-wall carbon nanotube/Fe3O4 hybrid materials. New Carbon Mater 2013;28(3):184-190.

[19] Wang L, Li J, Liu Y. Preparation of electromagnetic shielding wood-metal composite by electroless nickel plating. J For Res 2006;17(1):53-56.

[20] Mihut DM, Lozano K, Tidrow SC, Garcia H. Electromagnetic interference shielding effectiveness of nanoreinforced polymer composites deposited with conductive metallic thin films. Thin Solid Films 2012;520(21):6547-6550.

[21] Joshi A, Bajaj A, Singh R, Anand A, Alegaonkar PS, Datar S. Processing of graphene nanoribbon based hybrid composite for electromagnetic shielding. Composites Part B 2015;69:472-477.

[22] Chen Y, Li Y, Chu BTT, Kuo I, Yip M, Tai N. Porous composites coated with hybrid nano carbon materials perform excellent electromagnetic interference shielding. Composites Part B 2015;70:231237.

[23] Sun L, Li J, Wang L. Electromagnetic interference shielding material from electroless copper plating on birch veneer. Wood Sci Technol 2012;46(6):1061-1071.

[24] Lee BO, Woo WJ, Park HS, Hahm HS, Wu JP, Kim MS. Influence of aspect ratio and skin effect on EMI shielding of coating materials fabricated with carbon nanofiber/PVDF. J Mater Sci 2002;37(9):1839-1843.

[25] Wang L, Li J, Liu Y. Surface characteristics of electroless nickel plated electromagnetic shielding wood veneer. J For Res 2005;16(3):233-236.

[26] Hui B, Li J, Wang L. Electromagnetic shielding wood-based composite from electroless plating corrosion-resistant Ni-Cu-P coatings on Fraxinus mandshurica veneer. Wood Sci Technol 2014;48(5):961-979.

[27] Lin C, Lin Z, Lou C, Kuo T, Lin J. Wood plastic composites: Using carbon fiber to create electromagnetic shielding effectiveness. J Thermoplast Compos Mater 2015;28(7):1047-1057. 
[28] Oka H, Uchidate S, Sekino N, Namizaki Y, Kubota K, Osada H, Dawson FP, Lavers JD. Electromagnetic Wave Absorption Characteristics of Half Carbonized Powder-Type Magnetic Wood. IEEE Trans Magn 2011;47(10):3078-3080.

[29] Ding Z, Shi SQ, Zhang H, Cai L. Electromagnetic shielding properties of iron oxide impregnated kenaf bast fiberboard. Composites Part B 2015;78:266-271.

[30] Makoviny I, Makovinyiova K. Shielding of electromagnetic radiation by using wood-cement boards modified with carbon in microwave frequency band. Eur J Wood Wood Prod 2011;69(4):671673.

[31] Jalali M, Dauterstedt S, Michaud A, Wuthrich R. Electromagnetic shielding of polymer-matrix composites with metallic nanoparticles. Composites Part B 2011;42(6):1420-1426.

[32] Xia C, Ren H, Shi SQ, Zhang H, Cheng J, Cai L, Chen K, Tan H. Natural fiber composites with EMI shielding function fabricated using VARTM and $\mathrm{Cu}$ film magnetron sputtering. Appl Surf Sci 2016;362:335-340.

[33] Xia C, Zhang S, Ren H, Shi SQ, Zhang H, Cai L, Li J. Scalable fabrication of natural-fiber reinforced composites with electromagnetic interference shielding properties by incorporating powdered activated carbon. Materials 2016;9(1):10.

[34] Merk V, Chanana M, Gierlinger N, Hirt AM, Burgert I. Hybrid wood materials with magnetic anisotropy dictated by the hierarchical cell structure. ACS Appl Mater Interfaces 2014;6(12):9760-9767.

[35] Cornell RM, Schwertmann U. The iron oxides: structure, properties, reactions, occurrences and uses. 2006, John Wiley \& Sons.

[36] Oka H, Terui M, Osada H, Sekino N, Namizaki Y, Oka H, Dawson FP. Electromagnetic wave absorption characteristics adjustment method of recycled powder-type magnetic wood for use as a building material. IEEE Trans Magn 2012;48(11):3498-3500.

[37] Oka H, Hojo A, Osada H, Namizaki Y, Taniuchi H. Manufacturing methods and magnetic characteristics of magnetic wood. J Magn Magn Mater 2004;272:2332-2334.

[38] Oka H, Hojo A, Seki K, Takashiba T. Wood construction and magnetic characteristics of impregnated type magnetic wood. J Magn Magn Mater 2002;239(1-3):617-619.

[39] Chen J, Zhao D, Ge H, Wang J. Graphene oxide-deposited carbon fiber/cement composites for electromagnetic interference shielding application. Constr Build Mater 2015;84:66-72.

[40] Li P, Zhu AM, Liu QL, Zhang QG. Fe3O4/poly(N-Isopropylacrylamide)/chitosan composite microspheres with multiresponsive properties. Ind Eng Chem Res 2008;47(20):7700-7706.

[41] Gao HL, Wu GY, Guan HT, Zhang GL. In situ preparation and magnetic properties of Fe3O4/wood composite. Mater Technol 2012;27(1):101-103. 
[42] Calvo S, Arias-Duque N, Giraldo O, Rosales-Rivera A. Compositional, thermal and magnetic analysis of bamboo fibers covered with magnetite nanoparticles by in-situ co-precipitation. Rev Mex Fis 2012;58(2):134-137.

[43] Xia C, Shi SQ, Cai L, Hua J. Property enhancement of kenaf fiber composites by means of vacuum-assisted resin transfer molding (VARTM). Holzforschung 2015;69(3):307-312.

[44] Islam MS, Hamdan S, Talib ZA, Ahmed AS, Rahman MR. Tropical wood polymer nanocomposite (WPNC): The impact of nanoclay on dynamic mechanical thermal properties. Composites Sci Technol 2012;72(16):1995-2001.

[45] Hristov V, Vasileva S. Dynamic mechanical and thermal properties of modified poly(propylene) wood fiber composites. Macromol Mater Eng 2003;288(10):798-806.

[46] Hazarika A, Mandal M, Maji TK. Dynamic mechanical analysis, biodegradability and thermal stability of wood polymer nanocomposites. Composites Part B 2014;60:568-576.

[47] Xia C, Shi SQ, Cai L. Vacuum-assisted resin infusion (VARI) and hot pressing for $\mathrm{CaCO}_{3}$ nanoparticle treated kenaf fiber reinforced composites. Composites Part B 2015;78:138-143.

[48] Hu Z, Zen X, Gong J, Deng Y. Water resistance improvement of paper by superhydrophobic modification with microsized $\mathrm{CaCO}_{3}$ and fatty acid coating. Colloid Surface A 2009;351(1-3):65-70.

[49] Xu ZZ, Wang CC, Yang WL, Fu SK. Synthesis of superparamagnetic Fe3O4/SiO2 composite particles via sol-gel process based on inverse miniemulsion. J Mater Sci 2005;40(17):4667-4669.

[50] Iida H, Takayanagi K, Nakanishi T, Osaka T. Synthesis of Fe3O4 nanoparticles with various sizes and magnetic properties by controlled hydrolysis. J Colloid Interface Sci 2007;314(1):274-280. 